\section{Políticas de prevenção ao abuso de drogas no Brasil e nos Estados Unidos}

\author{
Public policies for drug abuse prevention in Brazil \\ and the United States
}

\section{Políticas de prevención para evitar el consumo \\ de drogas en Brasil y Estados Unidos}


O estabelecimento de políticas públicas está diretamente ligado ao modelo de relação instituído entre Estado e sociedade. Para Höfling 1, a definição de políticas públicas evidencia conflitos de interesse e arranjos produzidos nas esferas de poder que envolvem instituições do Estado e da sociedade.

Somente a partir do século XXI aspectos das conjunturas mundial e local passaram a permitir a configuração dos jovens como sujeitos de direitos 2. Esses aspectos incluíram, de um lado, os efeitos de políticas neoliberais desagregadoras, a violência do tráfico de drogas, o comércio de armas e a corrupção policial e, por outro lado, as novas formas de vivenciar a relação espaço-tempo e as criativas estratégias de inserção social. As políticas públicas elaboradas para a juventude no Brasil emergem nesse cenário, pautadas pelos movimentos sociais contra a exclusão e a estigmatização dos jovens e formalizadas somente há uma década, com a criação da Secretaria Nacional de Juventude em 2005.

A despeito dessa mudança de concepção das políticas públicas, o foco na juventude ainda permanece fortemente atrelado à ideia de risco e transgressão, tanto no pensamento científico como na opinião pública ${ }^{3}$. A negação da cidadania dos jovens está relacionada a uma percepção social de que a esse grupo devem ser destinadas ações repressivas para evitar os desvios do processo de socialização, como a delinquência e o uso de drogas, pressupostos comuns a políticas públicas historicamente destinadas à juventude 4. Dados da Secretaria Nacional de Juventude e do Ministério da Saúde evidenciam essa realidade ao apontarem que mais de $20 \%$ dos jovens brasileiros entre 15 e 24 anos não estudam nem trabalham 5, e 54,8\% da população carcerária do país é formada por jovens entre 18 e 29 anos 6 .

$\mathrm{Na}$ esteira desses pressupostos, se estabelece também o debate sobre o chamado "fenômeno das drogas” entre os jovens. Segundo Passos \& Souza 7, a Guerra às Drogas, instituída pelos Estados Unidos na década de 1970, tornou-se simultaneamente um meio de controle social e de ampliação da política neoliberal a partir de práticas de poder e violência fomentadas pela economia bélica e pela lógica de consumo. Do ponto de vista do controle da demanda e da oferta de drogas, porém, a ineficácia desse modelo repressivo é evidente 8 .

Dentre as políticas públicas adotadas na área de drogas mundialmente, como o controle da oferta e o acesso a serviços sociais e de saúde para usuários, a prevenção é a que apresenta a melhor relação custo-benefício para a redução tanto do consumo abusivo como de suas consequências. É estimado que, para cada dólar usado em programas de prevenção escolar, evita-se o gasto, em média, de 18 dólares com o custo social de problemas relacionados ao abuso de drogas 9 . Entretanto, como as demais políticas, sua implementação tem sido menos baseada em evidências científicas e mais no que os políticos consideram importante 10. Esse hiato produz divergências que dificultam a elaboração de uma política pública de prevenção integrada em termos de ações efetivas. Além disso, a pequena produção científica brasileira sobre prevenção ao abuso de drogas gera a importação de modelos de intervenção produzidos em outros contextos 11,12.

Este artigo pretende discutir o modelo de políticas públicas de prevenção ao abuso de drogas destinadas aos jovens no Brasil e nos Estados Unidos, em função da hegemonia estadunidense na determinação das políticas sobre drogas nos países dependentes. O percurso metodológico adotado se aproxima do modelo de avaliação do desenho da política. De acordo com o guia de avaliação de políticas públicas do Governo Federal do Brasil, "é recomendada a realização de avaliação de desenho como forma de verificar as hipóteses estabelecidas para a política quando da sua formulação, ou mesmo aprofundar pontos e definições que, na época, não se apoiavam em evidências claras para a sua adoção” 13 (p. 121). Segundo Jannuzzi 14, a avaliação de desenho envolve análises com base em dados secundários ou compilação de estudos, com maior ou menor abrangência, desenvolvidos a respeito do tema que a política abrange, dos determinantes da problemática em foco e dos programas e projetos elaborados para intervir em tais questões. Para tanto, serão abordadas as legislações vigentes, seus escopos e contexto de elaboração, os diferentes programas de prevenção e sua efetividade.

\section{O proibicionismo dos Estados Unidos e sua importação para o Brasil}

As políticas proibicionistas estadunidenses remetem ao movimento de temperança do final do século XIX e início do século XX. Estabelecido pela classe média, adotava uma ideologia de que o álcool supostamente gerava dependência, de modo inevitável e, por isso, destruía o caráter moral e a saúde física e mental dos seus consumidores. Os programas preventivos ao uso de drogas seguiram, 
pari passu, a política de abstinência compulsória, como a incorporação da "Instrução Científica da Temperança" ao currículo escolar 15.

Durante os anos 1980, o crescimento do conservadorismo político do governo do Presidente Ronald Reagan favoreceu o recrudescimento das políticas repressivas. Em 1988, foi promulgada a Lei Federal Antidrogas, que criou a Divisão de Política de Controle de Drogas (Office of National Drug Control Policy - ONDCP), visando a estabelecer prioridades para o controle de drogas. Simultaneamente ao reforço da política "just say no" (basta dizer não) dirigida aos jovens, porém, houve corte no orçamento para programas de prevenção ao uso de drogas 16 e, mais adiante, manipulação de dados estatísticos feita pela ONDCP para forjar a eficácia da Guerra às Drogas 17.

No Brasil, as políticas proibicionistas de controle social se adequaram ao modelo estadunidense desde o princípio. Segundo Torcato 18, o movimento de temperança brasileiro dos anos 1920 também pressionou os legisladores por uma política pública que condenasse o uso de bebidas alcoólicas, embora sem êxito na aprovação da abstinência compulsória. O aumento do consumo para fins recreacionais, e não mais exclusivamente terapêuticos, contudo, fez com que a classe médica buscasse a interdição desses usos e configurasse a droga como um problema de saúde pública. Esse grupo conseguiu, por meio da articulação política, a sanção da primeira lei específica sobre drogas no Brasil em 1921, sob forte influência da lei seca estadunidense 19.

O surgimento dessa lei e das subsequentes reafirmou, internamente, os tratados internacionais dos quais o Brasil é signatário. A Constituição Federal de 1988 valida o que deliberou a Convenção Contra o Tráfico Ilícito de Entorpecentes e de Substâncias Psicotrópicas nesse mesmo ano 20. Portanto, a análise da política pública nacional sobre drogas exige a compreensão do entrelaçamento entre o proibicionismo multilateral estabelecido pela Organização das Nações Unidas (ONU), a repressão militarizada dos Estados Unidos e os interesses das classes dominantes do Brasil 21.

\section{As políticas de prevenção ao abuso de drogas no Brasil e nos Estados Unidos}

Atualmente, as legislações que regem a política pública sobre controle de drogas nos Estados Unidos e no Brasil são, respectivamente, a Lei Federal Antidrogas de 198822 e a Lei no 11.343, de 23 de agosto de 2006, com as alterações trazidas pela Lei no 13.840, de 5 de junho de 2019 23. Vale ressaltar que os artigos que tratam da prevenção ao abuso de drogas permaneceram sem modificações. Ambas dispõem sobre medidas de controle tanto da oferta como da demanda, embora a ênfase recaia na repressão da oferta. As duas leis destinam uma seção específica à prevenção ao abuso de drogas, com especificidades em relação ao financiamento, execução e avaliação da política. A lei estadunidense detalha, ainda, o público alvo da política: estudantes, pessoas em situação de rua, comunidades e adolescentes em conflito com a lei. Este estudo terá como foco os estudantes, uma vez que a política de prevenção brasileira é fundada em ações voltadas a essa população.

\section{Legislação estadunidense de prevenção ao abuso de drogas}

A ONDCP prioriza os serviços de saúde mental voltados aos usuários em relação às estratégias educacionais de prevenção. Ou seja, a prioridade do governo estadunidense não é o controle da demanda e, dentro dela, não é a prevenção.

A Lei Antidrogas afirma que o locus privilegiado dos programas de prevenção deve ser a escola, que "claramente e consistentemente ensine que o uso de drogas ilícitas é errado e prejudicial" 22. Os programas devem ser ofertados para todos os níveis de ensino utilizando material didático próprio, elaborado em conformidade com o princípio de abstinência como meta exclusiva. $\mathrm{O}$ fato de as drogas serem consideradas como danosas em si estigmatiza o usuário como perigoso e potencialmente violento. A associação entre drogas e violência como uma relação de causa e efeito é um dos componentes centrais dos programas de prevenção.

A Lei Antidrogas determina também que os professores e profissionais de saúde mental e educação recebam formação prévia sobre abuso de drogas para executar atividades de prevenção. Visando a garantir a reprodução do discurso legalista, a estratégia usada pela política estaduni- 
dense é a de considerar que a condução dos programas de prevenção ao abuso de drogas é uma prerrogativa dos especialistas.

A seleção dos programas de prevenção a serem executados com financiamento do governo é realizada com base na relação custo-eficácia desses serviços. Há controvérsias, contudo, sobre os critérios estabelecidos para que os programas sejam considerados recomendáveis 24,25 .

\section{Financiamento das políticas de prevenção}

Segundo dados do orçamento nacional de controle às drogas dos Estados Unidos, dos cerca de 27 bilhões de dólares previstos para gastos nesse setor a cada ano do triênio de 2016 a 2018, em média $56,7 \%$ foram destinados à redução da oferta, ou seja, à repressão da produção e do tráfico de drogas, tanto nos Estados Unidos como internacionalmente ${ }^{26}$. Avaliados em conjunto, apenas uma média de $5 \%$ dos gastos com o controle da demanda e da oferta foram designados para programas de prevenção ao abuso de drogas. A Tabela 1 mostra a distribuição dos valores específicos divididos por área a que se destinam.

A análise dos gastos com ações de prevenção ao abuso de drogas entre 2009 e 2018 evidencia uma diminuição paulatina do orçamento nesse setor, em torno de 8,4\% 26 . A redução gradativa de verbas para ações de prevenção revela a falta de prioridade dada a programas de prevenção ao abuso de drogas e evidencia o primado da perspectiva de repressão às drogas.

\section{Programas de prevenção}

No final dos anos 1990, o governo passou a exigir que as ações preventivas desenvolvidas nas escolas se baseassem em evidências científicas. Desde então, para receber financiamento federal, os programas de prevenção devem se adequar a critérios de eficácia. As primeiras medidas de eficácia estabeleciam como objetivo inicial "educar e habilitar a juventude da América a rejeitar as drogas ilegais, bem como o álcool e o tabaco", esclarecendo que essa "estratégia tem como foco a juventude por questões morais e práticas" 27 (p. 38).

Atualmente, os critérios programáticos são centrados em aspectos metodológicos da pesquisa no campo da prevenção, embora a meta da abstinência permaneça. Desta forma, foram criadas várias listas de programas de prevenção baseados em evidências estabelecidas por diferentes órgãos federais e acadêmicos, e as escolas se baseiam nesses critérios para selecioná-los 28 .

Pesquisadores têm criticado o processo de avaliação dos programas e os critérios para considerá-los baseados em evidências científicas 29,30,31. Segundo Gandhi et al. 32, há poucos estudos com avaliação empírica, limitadas evidências de efetividade em longo prazo, ausência de avaliadores independentes e uso de subamostras, produzindo evidências com vieses. De acordo com Holder 33, o campo de pesquisa acerca de programas de prevenção nos Estados Unidos apresenta falta de transparência nas análises dos resultados, ausência de relato de dados negativos nas publicações e poucas replicações realizadas por pesquisadores não vinculados ao grupo que idealizou o programa. Gorman 34

Tabela 1

Valores do orçamento destinado ao controle da demanda e da oferta de drogas nos Estados Unidos.

\begin{tabular}{|c|c|c|c|c|c|}
\hline \multirow[t]{2}{*}{ Ano } & \multicolumn{2}{|c|}{ Controle da demanda (em bilhões de dólares) } & \multicolumn{3}{|c|}{ Controle da oferta (em bilhões de dólares) } \\
\hline & Prevenção & Tratamento & Cumprimento da lei & $\begin{array}{c}\text { Busca e apreensão } \\
\text { de drogas }\end{array}$ & Internacional \\
\hline 2016 & 1,5 & 9,8 & 9,3 & 4,7 & 1,5 \\
\hline 2017 & 1,5 & 10,6 & 9,3 & 4,6 & 1,5 \\
\hline 2018 & 1,3 & 10,8 & 9,2 & 5,0 & 1,4 \\
\hline Total & $4,3(5,24 \%)$ & $31,2(38,05 \%)$ & $27,8(33,90 \%)$ & $14,3(17,44 \%)$ & $4,4(5,37 \%)$ \\
\hline
\end{tabular}


questiona a perspectiva de ciência adotada pelos pesquisadores estadunidenses do campo da prevenção ao ter como objetivo único provar que os programas funcionam.

\section{Legislação brasileira de prevenção ao abuso de drogas}

A Secretaria Nacional de Políticas sobre Drogas (SENAD) é o órgão do Ministério da Justiça e Segurança Pública que coordena as ações de prevenção ao abuso de drogas, atenção e reinserção social de usuários, além da repressão ao tráfico. Foi criada em 1998, no governo do Presidente Fernando Henrique Cardoso, como Secretaria Nacional Antidrogas, com o intuito de ratificar, para a comunidade internacional, a adoção do modelo de repressão às drogas 35 .

A Lei no 11.343/2006, que rege a política pública sobre drogas, estabelece como um dos princípios da prevenção "o fortalecimento da autonomia e da responsabilidade individual" e preconiza também o "não-uso" ou o "retardamento do uso" e a redução de riscos como os objetivos almejados para ações preventivas. Com as recentes alterações trazidas pela Lei no 13.840/201923, no entanto, o sistema deixou de assumir a perspectiva da redução de danos, adotando a abstinência como única abordagem ao uso de drogas.

É estipulado que haja a implantação de programas de prevenção em instituições de ensino público e privado e, para tanto, os profissionais dos três níveis de ensino devem receber formação por meio de políticas de educação continuada. As pesquisas, no entanto, mostram o despreparo dos professores para o desempenho dessa função por medo, falta de informação ou de habilidade para abordar o tema 36,37 .

A Lei preconiza também que as atividades preventivas sejam baseadas em fundamentação científica, para evitar o preconceito e a estigmatização dos usuários e dos serviços. Pesquisas acerca da efetividade das intervenções preventivas no Brasil são escassas, pouco valorizadas e descontínuas 11,12,38 ou circunstanciadas a programas localizados e sem alcance nacional. A partir de 2013, porém, por iniciativa do Escritório das Nações Unidas sobre Drogas e Crimes (United Nations Office on Drugs and Crime - UNODC) e em parceria com os Ministérios da Saúde, do Desenvolvimento Social e Combate à Fome e pela SENAD, três programas originalmente desenvolvidos nos Estados Unidos estão sendo validados para o Brasil, como parte do pacote de exportação da "Guerra às Drogas” estadunidense: o jogo Elos, o \#Tamojunto e o Famílias Fortes.

A intersetorialidade e a corresponsabilização entre os serviços que ofertam atividades de prevenção e os que atuam com usuários e suas famílias são indicadas na lei. Para o estabelecimento de colaboração mútua, são sugeridas parcerias com instituições do setor privado e com os diversos segmentos sociais. Os movimentos sociais, em contrapartida, não aparecem como importantes parceiros. Atualmente, no entanto, são os movimentos sociais de saúde mental, associados às universidades, que têm exercido um papel protagonista de controle social das políticas públicas sobre drogas.

\section{Financiamento das políticas de prevenção}

Os Relatórios de Gestão da SENAD fazem o demonstrativo da execução orçamentária e financeira do programa Crack, É Possível Vencer, que fomenta a rubrica Prevenção ao Uso/Abuso de Drogas no Brasil com os seguintes objetivos: promover e articular ações continuadas de prevenção ao uso de drogas, de forma a informar, desestimular o uso inicial, incentivar a diminuição do consumo e diminuir os riscos e dados associados ao seu uso indevido 39,40,41. A Tabela 2 apresenta os dados dos três últimos relatórios divulgados pela SENAD (2015-2017), a partir do orçamento oriundo do Fundo Nacional Antidrogas (FUNAD).

Embora os valores efetivamente gastos com ações preventivas tenham dobrado entre 2015 e 2017 , o que se evidencia é a discrepância entre o que é orçado e a real despesa nesse setor. Oliveira 42 aponta uma fragilidade na transparência dos dados da SENAD, uma vez que não são dadas informações sobre o porquê dessa restrição de verbas, tampouco para a transferência de algumas ações da política de prevenção para outros ministérios.

Até 2013, o Governo Federal não dispunha de uma dotação orçamentária específica para a política de prevenção ao abuso de drogas. Ao dar início ao financiamento desta política, o fez por meio da 
Tabela 2

Dotação e despesa empenhada e liquidada destinadas à prevenção ao uso/abuso de drogas no Brasil.

\begin{tabular}{lccc}
\hline Ano & $\begin{array}{c}\text { Dotação (em milhões } \\
\text { de reais) }\end{array}$ & $\begin{array}{c}\text { Despesa (em milhões de reais) } \\
\text { Liquidada }\end{array}$ \\
\hline 2015 & 128.903 .543 & 46.342 .328 & 5.190 .116 \\
2016 & 115.915 .899 & 99.978 .678 & 13.985 .718 \\
2017 & 109.464 .928 & 87.994 .325 & 10.593 .491 \\
\hline
\end{tabular}

importação de tecnologia estadunidense e não por meio do fomento a uma linha de pesquisa nacional sobre o tema.

A Coordenação de Saúde Mental, Álcool e Outras Drogas, do Ministério da Saúde, realizou estudos de adaptação cultural de três programas de prevenção ao uso de drogas. Trata-se de programas de prevenção universais que foram elaborados originalmente nos Estados Unidos: Good Behavior Game [Jogo do Bom Corportamento] 43, Unplugged [Desplugado] 44 e Strengthening Family Program [Programa Fortalecimento da Família - SFP] 45. Dados requeridos ao Serviço de Informação ao Cidadão do Governo Federal referem que, entre 2013 e 2014, foram gastos R\$ 2.230.020,19 pelo Ministério da Saúde com pagamento a três instituições internacionais detentoras dos direitos autorais dos programas (Oxford Brookes University - Reino Unido; University College Ghent - Bélgica; e American Institutes for Research - Estados Unidos) para aquisição destes e acompanhamento das intervenções-piloto em dez municípios de dois estados brasileiros, bem como para contratação de serviços de tradução dos materiais didáticos originais, ilustração, diagramação, dublagem, além de impressão e distribuição para os territórios e pagamento de equipe técnica para supervisionar a execução dos programas junto ao Ministério da Saúde.

A implementação dos três programas está sob coordenação de pesquisadores da Universidade Federal de São Paulo (UNIFESP) e da Universidade Federal de Santa Catarina (UFSC). No período entre 2013 e 2014 foram destinadas a essas universidades, respectivamente, R\$ 682.866,40 e R\$299.979,20. De 2015 em diante, a Coordenação de Saúde Mental, Álcool e Outras Drogas informa que os gastos têm sido menores em função de que a aquisição dos programas foi feita uma única vez.

\section{Programas de prevenção brasileiros}

A história dos programas de prevenção ao consumo de drogas no Brasil é marcada pela importação de modelos de outras realidades. Canoletti \& Soares 11 consideram que há duas fases das ações preventivas no Brasil. A primeira fase, que se estende até o início da década de 1990, envolve a ausência de investigações científicas na área, ações de prevenção esparsas e descontínuas e programas inadequados elaborados em outros países. A segunda fase ocorre pós-epidemia da aids por meio dos programas de prevenção à transmissão do HIV pelo compartilhamento de materiais para uso de drogas injetáveis. Nessa fase, em função de investimentos da Organização das Nações Unidas para a Educação, Ciência e Cultura (Unesco), os programas de prevenção ao uso de drogas passam a dar mais ênfase à educação.

As ações oficiais de prevenção que tiveram alcance nacional e que se mantêm até os dias atuais são muito restritas. Resumem-se a cursos de capacitação à distância, como por exemplo o Sistema para Detecção do Uso Abusivo e Dependência de Substâncias Psicoativas: Encaminhamento, Intervenção Breve, Reinserção Social e Acompanhamento (SUPERA) 46, além de um programa de prevenção, o Programa Educacional de Resistência às Drogas (PROERD), desenvolvido nas escolas pela Polícia Militar desde 1992. O PROERD é proveniente do programa Drug Abuse Resistance Education (DARE), criado pelo Departamento de Polícia de Los Angeles (Estados Unidos) em 1983 para crianças do Ensino Fundamental. Embora o PROERD continue a ser ofertado nas escolas, há pesquisas que datam dos anos 1990 10,47,48 mostrando a ineficácia do DARE. Da mesma forma, estudos atuais avaliaram negativamente o PROERD, tanto em função da utilização de estratégias de amedrontamento 49 , como pela ausência de evidências de eficácia 50. 
Os programas de prevenção ao abuso de drogas atualmente implementados no Brasil foram renomeados a partir dos que lhes serviram de modelo. O Quadro 1 apresenta os programas brasileiros e os respectivos programas originalmente desenvolvidos nos Estados Unidos.

Tanto o programa Unplugged, adaptado por um grupo de pesquisadores europeus 51 a partir de um modelo de prevenção tradicional dos Estados Unidos 52, como o SFP são baseados no Modelo de Influência Social Global 53, que envolve o ensino de habilidades de vida para que o estudante evite as influências sociais pró-drogas, principalmente dos pares, e recuse o uso de drogas, ainda nos moldes do "just say no" do auge da Guerra às Drogas. O Good Behavior Game nasce com objetivos diferentes na década de 1960. A finalidade é melhorar a qualidade da sociabilidade entre os alunos e entre eles e o professor, especialmente em salas de aula onde há crianças com problemas de comportamento relacionados à interação social. O foco central não é, portanto, a prevenção ao uso de drogas. Estudos têm mostrado, porém, que, ao interferir positivamente na trajetória de desenvolvimento das crianças, elas tendem a se tornar adolescentes e adultos menos vulneráveis a comportamentos de risco, incluindo o abuso de drogas 54,55 .

Recentemente, o Ministério da Saúde, em parceria com a UNIFESP, lançou relatório com os dados acerca do processo de adaptação cultural e de avaliação de eficácia dos três programas sugeridos pelo UNODC 56. Tanto o programa Elos quanto o \#Tamojunto foram considerados inócuos, uma vez que os efeitos positivos não se mantiveram a longo prazo, e também iatrogênicos, pois produziram efeitos negativos.

Dentre os efeitos adversos, houve o aumento da agressividade e da disruptividade entre os estudantes avaliados como cooperativos no início do programa Elos, sendo que eles compunham a maioria da amostra do grupo que recebeu a intervenção 56 . No programa \#Tamojunto, não houve efeito significativo no consumo das drogas avaliadas (tabaco, maconha, cocaína, crack e beber em binge), além de ter ocorrido aumento de $30 \%$ de chance de iniciação ao uso de álcool durante o segmento de nove meses após a aplicação do programa 57.

A partir desses resultados, a SENAD, em articulação com a Coordenação Geral de Saúde Mental do Ministério da Saúde, suspendeu a implementação em larga escala dos programas \#Tamojunto, Elos e Famílias Fortes. Em função do que considerou como uma estratégia "arriscada", ou seja, a aplicação dos programas de prevenção em vários municípios brasileiros sem projetos-piloto, a SENAD estabeleceu uma nova condição para o financiamento de ações de prevenção. O inciso IV do art. 1o da resolução do CONAD no 1/2018 prevê que "o fomento e incentivo aos programas de prevenção próprios ou adaptados à realidade brasileira em articulação com organismos internacionais devem ser direcionados exclusivamente às iniciativas cujos resultados de impacto sejam satisfatoriamente mensuráveis no cumprimento dos objetivos de proteção" 41 (p. 128).

O Ministério da Educação não aprovou esses programas de prevenção e nem participa da sua implantação em função de terem origem em outros países e apresentarem proposta metodológica diferente da Política Nacional de Educação.

\section{Quadro 1}

Programas de prevenção brasileiros e seus originais.

\begin{tabular}{|l|c|}
\hline PROGRAMAS ORIGINAIS & PROGRAMAS BRASILEIROS \\
\hline Good Behavior Game & Jogo Elos (construindo coletivos - para crianças e 6 a 10 anos) \\
\hline Unplugged & \#Tamojunto (para adolescentes de 10 a 14 anos) \\
\hline Strengthening Families Program: for parents and youth 10-14 (SFP 10-14) & Famílias Fortes (para famílias e adolescentes de 10 a 14 anos) \\
\hline
\end{tabular}




\section{Análise comparativa das políticas de prevenção}

A América Latina depara-se com índices alarmantes de violência, relacionados com a internacionalização do crime, o qual é mantido pelo tráfico de armas e de drogas e pelas transferências ilegais de dinheiro 58, em grande medida criadas pela Guerra às Drogas. Esse cenário promoveu uma crescente resistência de alguns Estados latino-americanos contra o modelo mundial proibicionista de regulação às drogas, calcado quase que exclusivamente no controle da oferta. Esses Estados estabeleceram políticas sobre drogas alternativas e exigiram mudança do paradigma das abordagens repressivas para intervenções preventivas que tenham foco na redução de danos e na segurança cidadã 59 .

A construção de políticas sociais na América Latina, todavia, permanece dependente das políticas adotadas nos países centrais 60 . A relação de dependência entre países do primeiro e do terceiro mundo marca a produção do conhecimento que, em tese, subsidiaria as políticas públicas dos últimos. Essa dependência divide as nações que criam os chamados países centrais (como os Estados Unidos) daquelas que reproduzem modelos, ideias, tecnologia e cultura (os chamados países periféricos, como o Brasil). A produção dos saberes fica sob domínio das nações centrais, cabendo às nações periféricas o papel de consumidoras do conteúdo concebido no hemisfério norte 61. Essa relação tem conformado a política de prevenção ao abuso de drogas no Brasil. O Quadro 2 compara as características das políticas de prevenção ao uso de drogas nos Estados Unidos e no Brasil.

A despeito das significativas diferenças entre as políticas de controle às drogas adotadas pelos Estados Unidos e pelo Brasil, o paradigma repressor se impõe por meio da transferência de tecnologia em função da dominação político-econômica estadunidense. Mantém-se o modelo proibicionista, apenas deslocando o enfoque dos programas do amedrontamento para a persuasão.

A abordagem da redução de danos, não mais adotada pela política brasileira sobre drogas, parte do pressuposto de que, se não existe uma sociedade livre de drogas, é plausível lançar mão de estratégias para utilizá-las com o máximo de segurança possível, reconhecendo as diferentes possibilidades de uso - do recreacional, ocasional, frequente ao pesado - e, consequentemente, os distintos efeitos: benéficos, neutros ou prejudiciais 62 . Esta abordagem requer a substituição da perspectiva da prevenção para o da educação de crianças e adolescentes. Com base no princípio de que o consumo de drogas resulta de uma relação entre o sujeito, a droga e o ambiente, torna-se imprescindível a educação para a autonomia, para a proteção de si e da comunidade 63 . Os estudos que avaliam as práticas de cuidado aos usuários de drogas nos serviços de saúde brasileiros, tanto na atenção primária quanto na saúde mental, ainda se baseiam no modelo proibicionista. Essas práticas são pautadas em condutas estigmatizantes e moralizantes, além de apresentarem conhecimento insuficiente sobre a complexidade do tema das drogas 64,65.

Os interesses econômicos e políticos que determinam a legalidade ou ilegalidade das drogas se tornam evidentes tanto nos Estados Unidos quanto no Brasil, uma vez que os argumentos de proteção à

\section{Quadro 2}

Características das políticas de prevenção ao abuso de drogas nos Estados Unidos e no Brasil.

\begin{tabular}{|l|c|c|}
\hline CARACTERÍSTICAS & ESTADOS UNIDOS & BRASIL \\
\hline Legislação & Abstinência & Abstinência \\
\hline Financiamento & Investimento moderado & Baixo investimento \\
\hline Formulação da política de prevenção & $\begin{array}{c}\text { Embasada em pesquisas com avaliações } \\
\text { questionadas. Definidas majoritariamente pelo } \\
\text { próprio governo }\end{array}$ & $\begin{array}{c}\text { Importação de modelos estrangeiros. Definidas } \\
\text { majoritariamente pelo próprio governo }\end{array}$ \\
\hline Execução das ações & Organizações do Terceiro Setor & Grupos vinculados às universidades \\
\hline Resultado das intervenções & Moderado/Baixa eficácia & Moderado/Baixa eficácia \\
\hline
\end{tabular}


saúde dos sujeitos não se sustentam em função dos significativos danos individuais e sociais causados pelas drogas legais. As políticas públicas refletem esses interesses. A formulação da política de governo para a prevenção às drogas no Brasil tem-se estabelecido a partir de programas que são questionados em termos epistemológicos, teóricos e metodológicos 66,67,68,69.

Nos Estados Unidos, um país onde o sistema de saúde não é universal, o Terceiro Setor emerge como "justificativa ideológica do desengajamento do Estado em termos de ação pública" 70 (p. 11). As organizações não governamentais (ONGs) privadas sem fins lucrativos, predominantemente, executam os programas de prevenção ao uso de drogas.

No Brasil, embora o Sistema Único de Saúde (SUS) esteja expresso na Constituição e nas leis do Estado, garantir sua plena execução ainda é um dos maiores desafios do país. Teixeira \& Paim 71 apontam três estratégias de desmonte do SUS: a aprovação, pelos poderes Executivo e Legislativo, da participação do capital estrangeiro na saúde, terceirizações e planos de saúde; a defesa, por parte da mídia, dos políticos e de uma parcela da classe média, da criação de um SUS precário para pobres; e o subfinanciamento, por meio de uma das medidas mais drásticas de investida contra o SUS: a Emenda Constitucional 95/2016 (teto dos gastos). Incorporado ao subfinanciamento crônico do SUS, está o subfinanciamento da saúde mental 72. Entre 2001 e 2016, o Ministério da Saúde gastou, em média, 2,4\% do orçamento anual do SUS com saúde mental 73, quando o indicado pela Organização Mundial da Saúde (OMS) é de que os recursos destinados à saúde mental representem $5 \%$ do orçamento da saúde.

O financiamento da saúde mental foi reduzido em 0,8\% entre 2010 e 2016, e esse decréscimo está diretamente relacionado à implementação do programa Crack, É Possível Vencer em função da realocação dos gastos entre velhas e novas rubricas. Esse plano regulamenta o custeio das comunidades terapêuticas, um dos maiores gastos do programa, que se estabelece na contramão da Lei da Reforma Psiquiátrica por se configurar como um retorno ao modelo asilar e uma forma de privatização da saúde mental 72,74, além de não haver referências da eficácia da internação compulsória 75 .

O mapeamento das ações preventivas realizadas no Brasil é escasso. Abreu et al. 76 desenvolveram uma revisão sistemática com o objetivo de identificar os centros brasileiros que desenvolvem intervenções preventivas de forma sistemática e descrever estudos de avaliação dos programas produzidos. Os resultados desse estudo mostraram que os programas são executados por universidades públicas do Sudeste, com uma pequena parcela apoiada por agências de fomento à pesquisa. Ademais, verificaram que $62,79 \%$ dos programas acontecem em escolas, e apenas 6,82\% têm foco no uso de drogas. Concluem que as limitações metodológicas presentes no delineamento dos estudos demandam uma avaliação cautelosa dos dados de eficácia dos programas. O estudo de Laport et al. 77 mostrou que os profissionais da atenção primária que, em princípio, seriam os responsáveis pela prevenção e promoção de saúde no Brasil ainda possuem uma prática centrada na perspectiva curativista em relação às drogas, tanto em função de limitações da formação como da cultura organizacional estabelecida nas unidades de saúde brasileiras.

Para que uma política pública possa ser considerada efetiva, é necessário que ela seja sensível à conjuntura em que será adotada. Em um país com profundas desigualdades socioeconômicas, tal como o Brasil, os jovens não têm acesso a lazer, esporte, espaços de convivência social e, nesse contexto, a droga surge como uma das poucas opções de prazer. Sendo assim, persuadir os jovens a recusar as drogas parece não ser um caminho viável nesse cenário, ainda que por meio de estratégias interativas e informações realísticas sobre as drogas. São novos meios de manter velhas práticas.

As tensões entre os modelos proibicionista e de redução de danos se mantêm entre o que preveem as políticas de prevenção ao abuso de drogas da SENAD e do Ministério da Saúde e o que de fato é executado. Em decorrência disso, as ações de prevenção permanecem em meio ao embate entre o paradigma psicossocial e a perspectiva curativista e asilar de saúde mental. A Lei no 13.840/2019, ao aprofundar o aspecto repressor da Lei no 11.343/2006, tende a recrudescer os entraves à elaboração de programas educativos voltados para a promoção da reflexão e da responsabilidade dos jovens na relação com as drogas. 


\section{Considerações finais}

A Guerra às Drogas fracassou. Mais do que reconhecer as evidências que a ciência tem fornecido comprovando esse fato, é preciso acima de tudo superar a dependência brasileira do domínio estadunidense. Embora o Brasil seja signatário das convenções proibicionistas da ONU, a ineficácia dos programas preventivos, com resultados não significativos sobre a prevalência de uso entre os jovens, gera a necessidade de desconstruir a lógica persuasiva para elaborar a da educação.

Para Soares \& Jacobi 78, um programa de prevenção é definido não só pelo seu referencial teóricometodológico, mas também pelos seus pressupostos ideológicos. Portanto, cabe buscar autonomia em relação aos modelos de ações preventivas importados dos Estados Unidos, por meio de um debate mais amplo entre pesquisadores, profissionais, usuários e movimentos sociais, para o protagonismo de políticas mais adequadas ao Brasil, visando à construção de programas educativos que permitam a crianças e adolescentes brasileiros escolhas mais autênticas e que minimizem as suas condições de vulnerabilidade. Em que pesem todos os entraves citados neste estudo acerca dos programas brasileiros de prevenção ao abuso de drogas, há que se ressaltar a tentativa de elaborar uma política baseada em evidências científicas como um ganho inestimável para o país.

Este estudo busca contribuir com o debate sobre prevenção, atualmente em ascensão no país, analisando estratégias pertinentes e soberanas para uma política nacional sobre drogas que atenda à realidade brasileira. Apresenta limitações na medida em que os programas de prevenção selecionados se restringiram aos oferecidos a crianças e adolescentes em ambiente escolar. Pesquisas futuras podem investigar as análises de eficácia de diferentes programas de prevenção brasileiros já elaborados.

\section{Colaboradores}

D. I. B. Tatmatsu e C. E. Siqueira contribuíram na concepção e delineamento do estudo, análise e interpretação dos dados. Z. A. P. Del Prette contribuiu na revisão crítica do conteúdo intelectual do artigo. Todos os autores participaram da redação, aprovaram a versão final do manuscrito e se declaram responsáveis por todos os aspectos do trabalho, garantindo sua precisão e integridade.

\section{Informações adicionais}

ORCID: Daniely Ildegardes Brito Tatmatsu (00000003-2279-6997); Carlos Eduardo Siqueira (00000001-8993-3031); Zilda Aparecida Pereira Del Prette (0000-0002-0130-2911).

\section{Referências}

1. Höfling EM. Estado e políticas (públicas) sociais. Cad CEDES 2001; 21:30-41.

2. Novaes RCR. Prefácio. In: Castro JA, Aquino LM, Andrade CC, organizadores. Juventude e políticas sociais no Brasil. Brasília: Instituto de Pesquisa Econômica Aplicada; 2009. p. 13-22.

3. Takaeti BA, Vicentin MCG. A produção de conhecimento sobre juventude(s), vulnerabilidades e violências: uma análise da pós-graduação brasileira nas áreas de Psicologia e Saúde (1998-2008). Saúde Soc 2015; 24:945-63.

4. Sposito MP, Carano PCR. Juventude e políticas públicas no Brasil. Rev Bras Educ 2003; 24:16-39.

5. Ministério da Saúde. Diretrizes nacionais para a atenção integral à saúde de adolescentes e jovens na promoção, proteção e recuperação da saúde. Brasília: Ministério da Saúde; 2010.

6. Secretaria Geral da Presidência da República; Secretaria Nacional de Juventude. Mapa do encarceramento: os jovens do Brasil. Brasília: Presidência da República; 2015.

7. Passos EH, Souza TP. Redução de danos e saúde pública: construções alternativas à política de "Guerra às Drogas". Psicol Soc 2011; 23:154-62. 
8. Bokany V. Drogas no Brasil: entre a justiça e a saúde - proximidades e opiniões. São Paulo: Fundação Perseu Abramo; 2015.

9. U.S. Department of Health and Human Services. Substance abuse prevention dollars and cents: a cost-benefit analysis. Washington DC: U.S. Department of Health and Human Services; 2008.

10. Strang J, Babor T, Caulkins J, Fischer B, Foxcroft D, Humphreys K. Drug policy and the public good: evidence for effective interventions. Lancet 2012; 379:71-83.

11. Canoletti B, Soares CB. Programas de prevenção ao consumo de drogas no Brasil: uma análise da produção científica de 1991 a 2001. Interface (Botucatu) 2005; 9:115-29.

12. Noto AR, Galduróz JCF. O uso de drogas psicotrópicas e a prevenção no Brasil. Ciênc Saúde Colet 1999; 4:145-51.

13. Casa Civil, Presidência da República. Avaliação de políticas públicas: guia prático de análise ex post. Brasília: Presidência da República; 2018.

14. Jannuzzi PM. Avaliação de programas sociais: conceitos e referenciais de quem a realiza. Est Aval Educ 2014; 25:22-42.

15. Blocker Jr. JS. Did prohibition really work? Alcohol prohibition as a public health innovation. Am J Public Health 2006; 96:233-43.

16. Levine HG, Reinarman C. The trouble with drink and drugs: why prohibition and criminalization matter. Addiction 2010; 105:806-7.

17. Robinson MB, Scherlen RG. Lies, damned lies, and drug war statistics: a critical analysis of claims made by the office of National Drug Control Policy. New York: SUNY Press; 2014.

18. Torcato CEM. Breve história da proibição das drogas no Brasil: uma revisão. Inter-Legere 2014; 15:138-62.

19. Torcato CEM. O uso de drogas e a instauração do proibicionismo no Brasil. Saúde Transform Soc 2013; 4:117-25.

20. Ventura CAA. Drogas lícitas e ilícitas: do direito internacional à legislação brasileira. Rev Eletrônica Enferm 2011; 13:554-9.

21. Lima RCC. O problema das drogas no Brasil: Revisão legislativa nacional. Libertas 2010; 10:102-23.

22. United States. Public Law 100-690, November 18, 1988. Anti-Drug Abuse Act. Government Office 1988; 18 nov.

23. Brasil. Lei no 13.840 , de 5 de junho de 2019 . Altera as Leis no 11.343 , de 23 de agosto de 2006, 7.560, de 19 de dezembro de 1986, 9.250 , de 26 de dezembro de $1995,9.532$, de 10 de dezembro de 1997, 8.981, de 20 de janeiro de $1995,8.315$, de 23 de dezembro de 1991, 8.706 , de 14 de setembro de $1993,8.069$, de 13 de julho de 1990, 9.394, de 20 de dezembro de 1996, e 9.503, de 23 de setembro de 1997 , os Decretos-Lei no 4.048, de 22 de janeiro de 1942 , 8.621, de 10 de janeiro de 1946, e 5.452, de 1o de maio de 1943, para dispor sobre o Sistema Nacional de Políticas Públicas sobre Drogas e as condições de atenção aos usuários ou dependentes de drogas e para tratar do financiamento das políticas sobre drogas. Diário Oficial da União 2019; 6 jun.
24. Gorman DM. Has the National Registry of Evidence-based Program and Practices (NREPP) lost its way? Int J Drug Policy 2017; 45:40-1.

25. Gorman DM. The best of practices, the worst of practices: the making of science-based primary prevention programs. Psychiatr Serv 2003; 54:1087-9.

26. Office of the Presidente of the United States. National drug control budget. Washington DC: Office of the Presidente of the United States; 2018.

27. Office of National Drug Control Policy. National drug control strategy. Washington DC: Office of National Drug Control Policy; 1999.

28. Office of National Drug Control Policy. International standards on drug use prevention. Washington DC: Office of National Drug Control Policy; 2015.

29. Gorman DM. The decline effect in evaluations of the impact of the Strengthening Families Program for Youth 10-14 (SFP 10-14) on adolescent substance use. Child Youth Serv Rev 2017; 81:29-39.

30. Pape H. School-based programmes that seem to work: useful research on substance use prevention or suspicious stories of success? Nordic Alcohol Drugs 2009; 26:521-35.

31. McCambridge J. A case study of publication bias in an influential series of reviews of drug education. Drug Alcohol Rev 2007; 26:463-8.

32. Gandhi AG, Murphy-Graham E, Petrosino A, Chrismer SS, Weiss CH. The devil is in the details: examining the evidence for "proven" school-based drug abuse prevention programs. Eval Rev 2007; 31:43-74.

33. Holder H. Prevention programs in the $21 \mathrm{st}$ century: what we do not discuss in public. Addiction 2010; 105:578-81.

34. Gorman DM. Drug and violence prevention: rediscovering the critical rational dimension of evaluation research. J Exp Criminol 2005; $1: 39-62$.

35. Garcia MLT, Leal FX, Abreu CC. A política antidrogas brasileira: velhos dilemas. Psicol Soc 2008; 20:267-76.

36. Ferreira TCD, Sanchez ZM, Ribeiro LA, Oliveira LG, Nappo SA. Percepções e atitudes de professores e escolas públicas e privadas perante o tema drogas. Interface (Botucatu) 2010; 14:551-62.

37. Moreira FG, Silveira DX, Andreoli SB. Situações relacionadas ao uso indevido de drogas nas escolas públicas da cidade de São Paulo. Rev Saúde Pública 2006; 40:810-7.

38. Campos GM, Figlie NB. Prevenção ao uso nocivo de substâncias focada no indivíduo e no ambiente. In: Diehl A, Cordeiro DC, Laranjeira $\mathrm{R}$, organizadores. Dependência química: prevenção, tratamento e políticas públicas. Porto Alegre: Artmed; 2011. p. 481-94.

39. Secretaria Nacional de Políticas sobre Drogas, Ministério da Justiça. Relatório de gestão do exercício de 2015. Brasília: Conselho Nacional de Políticas sobre Drogas/Fundo Nacional Antidrogas; 2016. 
40. Secretaria Nacional de Políticas sobre Drogas, Ministério da Justiça. Relatório de gestão do exercício de 2016. Brasília: Conselho Nacional de Políticas sobre Drogas/Fundo Nacional Antidrogas; 2017.

41. Secretaria Nacional de Políticas sobre Drogas, Ministério da Justiça. Relatório de gestão do exercício de 2017. Brasília: Conselho Nacional de Políticas sobre Drogas/Fundo Nacional Antidrogas; 2018.

42. Oliveira $A B$. Fundo nacional antidrogas e o financiamento de programas e ações sobre drogas no Brasil no período de 2012 a 2015 [Monografia]. Brasília: Universidade de Brasília; 2017.

43. Barrish HH, Saunders M, Wolf MM. Good behavior game: effects of individual contingencies for group consequences on disruptive behavior in a classroom. J Appl Behav Anal 1969; 2:119-24.

44. Van der Keeft P, Wiborg G, Galanti M, Siliquini R, Bohrn K, Scatigna M, et al. 'Unplugged': a new European school programme against substance abuse. Drugs (Abingdon Engl) 2009; 16:167-81.

45. Kumpfer KL, Alvarado R, Whiteside H. Family-based interventions for substance abuse and prevention. Subst Use Misuse 2003; 38:175987.

46. Secretaria Nacional de Políticas sobre Drogas, Ministério da Justiça. Sistema para detecção do uso abusivo e dependência de substâncias psicoativas: encaminhamento, intervenção breve, reinserção social e acompanhamento. Módulo 5: atenção integral na rede de saúde. Brasília: Secretaria Nacional de Políticas sobre Drogas, Ministério da Justiça; 2016.

47. West SL, O’Neal KK. Project D.A.R.E. Outcome Effectiveness Revisited. Am J Public Health 2004; 94:1027-9.

48. Ennett ST, Tobler NS, Ringwalt CL, Flewelling RL. How effective is drug abuse resistense education? A meta-analysis of project DARE outcome evaluations. Am J Public Health 1994; 84:1394-401.

49. Domingues VG. Educação e guerra às drogas: uma reflexão sobre o PROERD na escola. Alabastro 2016; 1:56-72.

50. Caputi TL, McLellan AT. Truth and D.A.R.E.: Is D.A.R.E.'s new Keepin' it REAL curriculum suitable for American nationwide implementation? Drugs 2016; 24:49-57.

51. Faggiano F, Galanti MR, Bohrn K, Burkhart G, Vigna-Taglianti F, Cuomo L, et al. The effectiveness of a school-based substance abuse prevention program: EU-Dap cluster randomised controlled trial. Prev Med 2008; 47:537-43.

52. Botvin G, Griffin K. Life skills training: empirical findings and future directions. J Prim Prev 2004; 25:211-32.

53. Sussman S, Earlywine M, Wills T, Cody C, Biglan T, Dent C, Newcomb MD. The motivation, skills, and decision-making model of "drug abuse" prevention. Subst Use Misuse 2004; 39:1971-2016.
54. Kellam SG, Brown CH, Poduska JM, Ialongo NS, Wang W, Toyinbo P, et al. Effects of a universal clasroom behavior management program in first and second grades on young adult behavioral, psychiatric and social outcomes. Drug Alcohol Depend 2008; 95 Suppl 1:S5-28.

55. Kellam SG, Wang W, Mackenzie ACL, Brown $\mathrm{H}$, Ompad DC, Or F, et al. The impact of the Good Behavior Game, a universal classroombased preventive intervention in first and second grades, on high-risk sexual behaviors and drug abuse and dependence disorders into young adulthood. Prev Sci 2010; 15:6-18.

56. Ministério da Saúde; Universidade Federal de São Paulo. Prevenção ao uso de drogas: implantação e avaliação de programas no Brasil. Brasília: Ministério da Saúde; 2018.

57. Sanchez ZM, Valente Y, Sanudo A, Pereira APD, Cruz JI, Schneider DR, et al. The \#Tamojunto drug prevention program in Brazilian schools: a randomized controlled trial. Prev Sci 2017; 18:772-82.

58. Barreira C, Batista E. (In)Segurança e sociedade: treze lições. Fortaleza: Fundação Demócrito Rocha; 2011.

59. Carvalho IS. O despertar da América Latina: uma revisão do novo debate sobre políticas de drogas. Rio de Janeiro: Instituto Igarapé; 2014.

60. Paiva BA, Ouriques ND. Uma perspectiva latino-americana para as políticas sociais: quão distante está o horizonte? Katálysis 2006; 9:166-75.

61. Vecchio R. Ensino superior e a produção de conhecimento no Brasil e na América Latina. REBELA 2012; 2:24-36.

62. Feffermann M, Figueiredo R. Redução de danos como estratégia de prevenção de drogas entre jovens. Bol Inst Saúde 2006; 12:37-40.

63. Acselrad G. Avessos do prazer: drogas, aids e direitos humanos. 2a Ed. Rio de Janeiro: Editora Fiocruz; 2005.

64. Malvezzi CD, Nascimento JL. Cuidado aos usuários de álcool na atenção primária: moralismo, criminalização e teorias da abstinência. Trab Educ Saúde 2018; 16:1095-112.

65. Carvalho B, Dimenstein M. Análise do discurso sobre redução de danos num CAPSad III e em uma Comunidade Terapêutica. Temas Psicol (Online) 2017; 25:647-60.

66. Gorman DM. Hypothesis testing and the EUDap evaluation of the Unplugged curriculum. Prev Med 2009; 48:604-5.

67. Foxcroft DR, Callen H, Davies EL, OkuliczKozaryn K. Effectiveness of the strengthening families programme 10-14 in Poland: cluster randomized controlled trial. Eur J Public Health 2017; 27:494-500.

68. Riesch SK, Brown RL, Wang K, Canty-Mitchell J, Johnson DL. Strengthening Families Program (10-14): effects on the family environment. West J Nur Res 2012; 34:340-76.

69. Gorman DM, Conde E, Huber JC. The creation of evidence in 'evidence-based' drug prevention: a critique of the Strengthening Families Program plus life skills training evaluation. Drug Alcohol Rev 2007; 26:585-93. 
70. França Filho GC. Terceiro setor, economia social, economia solidária e economia popular: traçando fronteiras conceituais. Bahia: Análise \& Dados 2002; 12:9-19.

71. Teixeira CFS, Paim JS. A crise mundial de 2008 e o golpe do capital na política de saúde no Brasil. Saúde Debate 2018; 42:11-21.

72. Trapé TL, Onocko-Campos R. Modelo de atenção à saúde mental do Brasil: análise do financiamento, governança e mecanismos de avaliação. Rev Saúde Pública 2017; 51:19.

73. Oliveira EFA. Gastos da política de saúde mental e os rumos da Reforma Psiquiátrica [Tese de Doutorado]. Vitória: Universidade Federal do Espírito Santo; 2017.

74. Garcia ML, Oliveira EF. An analysis of the federal funding for mental health care in Brazil. Soc Work Health Care 2017; 56:169-88.
75. Werb D, Kamarulzaman A, Meacham MC, Rafful D, Fischer B, Strathdee SA, et al. The effectiveness of compulsory drug treatment: a systematic review. Int J Drug Policy 2016; 28:1-9.

76. Abreu S, Miranda APV, Murta SG. Programas preventivos brasileiros: quem faz e como é feita a prevenção em saúde mental? Psico USF 2016; 21:163-77.

77. Lapot TJ, Costa PHA, Mota DCB, Ronzani TM. Percepções e práticas dos profissionais da atenção primária à saúde na abordagem sobre drogas. Psicol Teor Pesq 2016; 32:143-50.

78. Soares CB, Jacobi PR. Adolescentes, drogas e Aids: avaliação de um programa de prevenção escolar. Cad Pesqui 2000; 109:213-37.

\section{Resumen}

El objetivo de este estudio fue discutir las politicas públicas de prevención del consumo de drogas destinadas a jóvenes en Brasil y Estados Unidos. La formulación de estas politicas en Brasil se ha establecido a partir de programas que son cuestionados internacionalmente, en términos epistemológicos, teóricos y metodológicos. Se concluye que la construcción de politicas sociales en Latinoamérica permanece influenciada por la continua dependencia de los países más importantes, ya que las tensiones entre los modelos prohibicionista y de reducción de daños se mantienen entre lo que prevén las politicas de prevención contra el consumo de drogas de la Secretaría Nacional de Politicas sobre Drogas y del Ministerio de Salud, y lo que de hecho se ejecuta. Se sugiere buscar autonomía respecto a los modelos de acciones preventivas, impuestos por parte de los países dominantes, mediante un debate más amplio entre investigadores, profesionales, consumidores y movimientos sociales para el protagonismo de políticas más adecuadas en Brasil.

Política Pública; Prevención; Abuso de Sustancias
Recebido em 04/Mar/2018

Versão final reapresentada em 30/Ago/2019

Aprovado em 07/Out/2019 